\title{
Quantum gases and quantum coherence
}

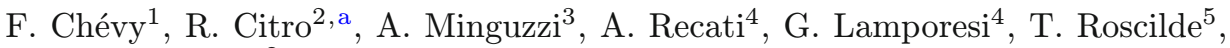 \\ and F. Illuminati ${ }^{2}$ \\ 1 ENS, Paris, France \\ 2 UniSa, Salerno, Italy \\ 3 LPMMC, Grenoble, France \\ 4 INO-CNR BEC, Trento, Italy \\ 5 ENS, Lyon, France
}

Received 16 May 2017

Published online 26 July 2017

This volume is a collection of papers connected to the International Workshop BEC2016 held at the University of Salerno (Italy) from August 31st to September 3rd, 2016. It is dedicated to the theoretical and experimental challenges in the field of quantum gases and quantum coherence, with strong links to other areas of physics including strongly correlated systems, out-of-equilibrium systems, synthetic gauge fields, disorder effects, dipolar gases, quantum information, etc. One of the main goals of this workshop consists in bringing together young researchers from Europe and overseas.

Since the observation of Bose-Einstein condensation (BEC) in 1995 and of superfluidity in atomic Fermi gases, ultracold quantum gases have become a very versatile testing ground for quantum many-body theories. The development of amazing experimental techniques allows for an unprecedented control of quantum gases, such as changing the dimensionality of the system, introducing disorder in a controlled way, and creating a whole variety of strongly correlated states. This has led to stronger and stronger connections between the physics of ultracold quantum gases and condensedmatter physics. This connection has been especially taken into consideration in the selection of contributions for the conference. In addition, attention has also been given to the recent activities in the realization of artificial gauge fields and synthetic dimensions, as well as the realization of BEC in other condensed matter systems (e.g. polaritons), which create further links to the condensed matter community.

A central point of contact between the cold-gas community and the condensedmatter community is offered by the study of superfluidity, which, in contrast to $\mathrm{He} 4$, can be realized by cold atoms in the regime of weak interactions. In such a regime the healing length of the system can become very large, significantly enhancing the size of the core of vortex excitations, and making the microscopic details of such excitations accessible to experiments. The manuscript (N. Verhelst et al.) builds upon these aspects to investigate theoretically the excitation modes of vortex cores - and in

\footnotetext{
a e-mail: citro@sa.infn.it
} 
particular the less studied radial mode - which are of central importance in the regime of quantum turbulence. The lively interface between condensed matter and ultracold quantum gases is further exemplified by the manuscript (L. Barbiero et al.), which focuses on the fermionic Hubbard model, namely the minimal model of strong correlations in electronic systems, which is now realized in several experiments on ultracold fermions in optical lattices. In particular most recent experiments have demonstrated access to non-local order parameter (so-called string order parameter) thanks to the remarkable developments of quantum-gas microscopy. This breakthrough inspires detailed studies of the role of such order parameters in the determination of the complex phase diagram of the extended Hubbard model - as offered by the present manuscript - comprising a variety of phases with gapped charge excitations and/or gapped spin excitations which are elucidated by corresponding charge and spin string order parameters. In the context of strong correlations and magnetism a future avenue is opened by the realization of long-range interacting systems with ultracold magnetic atoms. The manuscript (E. Lucioni et al.) reports on the progress made by the Pisa-Florence team to realize degenerate quantum gases of Dy - the element with the largest intrinsic magnetic moment among those which have been brought to quantum degeneracy in experiments so far. The dipolar interactions among the Dy atoms are a potential source of new phenomena, including significant off-site interactions for spin-polarized particles on a lattice (which characterize extended Hubbard models), or long-range spin-spin interactions for unpolarized particles which may be both itinerant or localized.

Another topic of much current interest is the non-equilibrium dynamics of lowdimensional quantum systems. Research on this topic is mainly motivated by ultracold gases experiments, where the unique degree of tunability, isolation, and long coherent times have enabled the exploration of phenomena not previously accessible in condensed matter experiments. In the latter, a strong coupling to the environment, combined with the short time scales associated to their microscopic properties, usually lead to rapid decoherence. Driving cold gases with time-dependent potentials can be used to probe several properties of interest, such as the relaxation dynamics. This is done in the manuscript (L. Foini \& T. Giamarchi) where the non-equilibrium dynamics of two tunnel coupled bosonic gases, which are created from the coherent splitting of a one-dimensional gas, is considered. The consequences of the tunneling are investigated and compared with equilibrium results. In particular, within a semiclassical approximation, correlation functions for the relative phase which are experimentally measurable are calculated and a transient regime displaying oscillations as a function of the distance is shown. However, the physics out of equilibrium is far richer than that. Optical lattices can be used to understand the properties of quantum transport in $1 \mathrm{D}$ systems, an example being the quantum pump. Topological quantum pumps are topologically equivalent to the quantum Hall state: In these systems, the charge pumped during each pumping cycle is quantized and coincides with the Chern invariant. However, differently from quantum Hall insulators, quantum pumps can exhibit novel phenomena such as the fractional quantization of the charge transport, as a consequence of their distinctive symmetries in parameter space. In the manuscript (P. Marra \& R. Citro) the fractional quantization of the charge and spin transport in a topological pump realized in a one-dimensional lattice via a periodically modulated Zeeman field is reported. In the proposed model, which is a spinful generalization of the Harper-Hofstadter model, the amount of spin current pumped during well-defined fractions of the pumping cycle is quantized as fractions of the spin Chern number. In the manuscript (Yi Zheng and Shi-Jie Yang) the quantum tunneling of correlated atoms in an optical lattice has been investigated in the presence of ac-driving fields. The effective Hamiltonian with density-dependent hopping rates has been deduced 
and it has been shown how the migration of correlated atoms in $1 \mathrm{D}$ and $2 \mathrm{D}$ lattices can be realized. Based on this mechanism, the authors propose a method to create two-partite entanglement. This model of out-of-equilibrium physics may provide applications in quantum computing and quantum information in ultracold atoms.

In the non-equilibrium context, quantum phase slips, i.e, the primary excitations in one-dimensional superfluids at low temperature, have been well characterized in most condensed-matter systems, with the notable exception of ultracold quantum gases. The manuscript (S. Simona et al.) presents the experimental investigation of the decay of supercurrents in one-dimensional Bose superfluids flowing along a periodic potential, which show signatures of the presence of quantum phase slips. In particular, by controlling the velocity of the superfluid and the interaction between the bosons the authors demonstrate the ability to drive a crossover from a regime of thermal phase slips into a regime of quantum phase slips. Achieving a good control of quantum phase slips in ultracold quantum gases requires to keep under control other phenomena such as the breaking of superfluidity at the critical velocity or the appearance of a Mott insulator in the strongly correlated regime. In the manuscript a clear description of current results along these directions is presented.

The controlled realization of paradigmatic quantum models via ultracold-atom experiments (or Feynman's analog quantum simulation) may transcend the field of low-energy physics and aim at relevant models of high energy physics. The manuscript (A. Celi) aims precisely at this goal, discussing how properly engineered honeycomb optical lattices with spatially dependent hopping terms can realize the physics of the Dirac's equation in Rindler coordinates - namely in an accelerated frame. This opens the charming possibility of implementing models of relativistic quantum field theory in accelerated frames using low-energy setups.

Two further manuscripts witness important developments in the study of quantum phenomena in quantum optics - whose conceptual framework still offers a fundamental reference for the cold-atom field. The manuscript (N. Bartolo et al.) discusses the generation of photonic Schrödinger's cat states in optical cavities via two-photon pumping, and the feedback offered on the quantum state by two different detection schemes (homodyning vs. photon counting) generating widely different quantum trajectories in potential experiments. Finally the manuscript (T. Zalialiutdinov et al.) explores N-photon transitions between atomic levels, and the associated (spinstatistics) selection rules, dictating the atom-light interactions in a regime in which an atomic transition is nearly resonant with a multiple $(\mathrm{N})$ of the light frequency.

A special contribution to this volume is represented by the manuscript by $M$. Blasone. Apparently disconnected from the physics of cold atoms, but strongly linked to quantum coherence, the author proposes the use of specific dynamical processes and, more in general of ideas from Physics, to model the evolution in time of musical structures. This approach is applied to two Études by F. Chopin, namely op.10 n.3 and op.25n.1, proposing some original description based on concepts of symmetry breaking/restoration and quantum coherence, which could be useful for interpretation. This nice analysis takes advantage of colored musical scores, obtained by implementing Scriabin's color code for sounds to musical notation and represents a travel through some topics of the conference via music.

Previous Editions of this workshop were held in Salerno (2001), Levico Terme (2003), Cortona (2005), Grenoble (2008), Nice (2010), Lyon (2012) and Levico Terme (2014). This Edition has been possible thanks to the support of Department of Physics \& Industrial Engineer (University of Salerno), the ERC project of Frederic Chévy, INO-CNR BEC, ESF, The European Physical Journal. A particular thank goes to Sabine Lehr, Agnès Henri and Nicolas Puyaubreau for their professional support. 
The Editors

Financially supported by

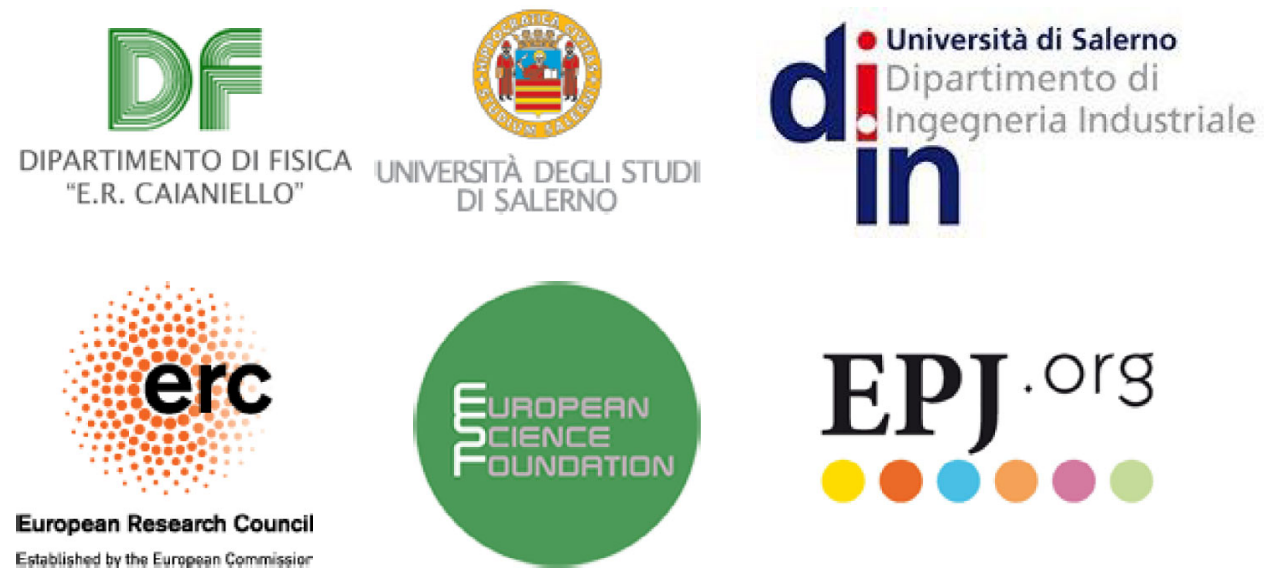

